\title{
Genetic Study of 12 SNPs involved in 11 Folate Metabolism Genes and Neural Tube Defects in Suzhou Children
}

\author{
Qin Zhu ${ }^{1 \#}$, Li Li ${ }^{2 \#}$, Ting Wang ${ }^{3}$, Wei Jiang ${ }^{4}$, Jie Ding ${ }^{3}$, Minjuan Liu ${ }^{5}$, Yun Wang ${ }^{1^{*}}$ and Haibo $\mathrm{Li}^{3^{*}}$ \\ ${ }^{1}$ Obstetrical Department, Nanjing Medical University Affiliated Suzhou Hospital, PR China \\ ${ }^{2}$ Obstetrical Department, Maternal and Children Health Care Hospital in Yinchuan, PR China \\ ${ }^{3}$ Center for Reproduction and Genetics, Nanjing Medical University Affiliated Suzhou Hospital, PR China \\ ${ }^{4}$ Center for Medical Ultrasound, Nanjing Medical University Affiliated Suzhou Hospital, PR China \\ ${ }^{5}$ Gynecology Department, Nanjing Medical University Affiliated Suzhou Hospital, PR China \\ \#Contributed equally and are joint first authors
}

"Corresponding authors: Haibo Li, Center for Reproduction and Genetics, Nanjing Medical University Affiliated Suzhou Hospital, 215002, PR China, Tel: 86051262362417; E-mail: lihaibo-775@163.com

Yun Wang, Obstetrical Department, Nanjing Medical University Affiliated Suzhou Hospital, 215002, PR China, Tel: 86051262362138; E-mail: wangyun_204@126.com

Received date: April 20, 2016; Accepted date: June 21, 2016; Published date: June 26, 2016

Copyright: ( 2016 Zhu Qin, et al. This is an open-access article distributed under the terms of the Creative Commons Attribution License, which permits unrestricted use, distribution, and reproduction in any medium, provided the original author and source are credited.

\begin{abstract}
Objective: Neural tube defect (NTD) incidence could be effectively reduced by folic acid supplementation before and during pregnancy. We studied single nucleotide polymorphisms (SNPs) involved in folate metabolism to explore genetic susceptibility to NTD. We studied the association between 12 SNPs involved in 11 folate metabolism genes and NTDs.
\end{abstract}

Methods: We enrolled 76 children with NTD and 188 control children. We genotyped 12 folate metabolism SNPs including CBS-C699T, DHFR-c594+59del19, GSTO1-C428T, MTHFD-G1958A, MTHFR-C677T, MTHFR-A1298C, MTR-A2756G, MTRR-A66G, NFE2L2-ins1+C11108T, RFC1-G80A, TCN2-C776T, and TYMS-1494del6 using SNaPShot genotyping technology and confirmed by Sanger sequencing.

Results: One SNP, TYMS-1494del6, and one compound wide-type genotype of RFC1-G80A, MTHFR-A1298C and TCN2-C776T might decrease NTD risk, and three compound mutation genotypes of MTHFD-G1958A, MTHFRC677T, and MTR-A2756G; MTHFD-G1958A, MTR-A2756G, and RFC1-G80A; and RFC1-G80A, MTHFR-A1298C, and TCN2-C776T might increase NTD risk. The TT genotype of TYMS-1494del6 $(P<0.001)$ and the AT+TT genotype of TYMS-1494del6 $(P=0.009)$ were significant genotypes. Fourteen in 188 control babies carried the compound wide-type genotype of RFC1-G80A, MTHFR-A1298C, and TCN2-C776T, but none in 76 NTD babies $(P=0.014)$. The ratios of the two compound mutants for MTHFD-G1958A, MTHFR-C677T, MTR-A2756G, and MTHFD-G1958A, RFC1-G80A, MTR-A2756G in NTD were higher than in control babies $(P=0.021)$ and RFC1G80A, MTHFR-A1298C, and TCN2-C776T $(P=0.029)$.

Conclusions: The TT genotype of TYMS-1494del6 and the two wide-type genotypes of RFC1-G80A, MTHFRA1298C, and TCN2-C776T are protective in NTD. Three compound mutation genotypes of MTHFD-G1958A, MTHFR-C677T, MTR-A2756G, MTHFD-G1958A, MTR-A2756G, RFC1-G80A, and RFC1-G80A, MTHFR-A1298C, TCN2-C776T might increase susceptibility to NTD.

Keywords: NTD; Folate; Folate metabolism genes; Single nucleotide polymorphisms

\section{Background}

Neural tube defects (NTDs) are a group of serious birth defects that affect the developing nervous system and include anencephaly, spina bifida, and encephalocele [1]. NTDs are the second most common of birth defects, which are preventable and are significant causes of infant death and childhood disability $[1,2]$. It is estimated that there are more than 300,000 NTDs worldwide each year, many of these occurring in low-resource countries [3]. Anencephaly and spina bifida are the most common NTDs and occur with about equal frequency, whereas encephalocele is seen less frequently. In anencephaly, there is partial or complete absence of the skull bones usually with a remnant of the brain, which is almost always fatal before or shortly after birth [4]. Spina bifida with meningomyelocele results from failure of the vertebral arches to close over an open neural tube defect, exposing the spinal cord and nerves [4]. Although most cases of spina bifida are open, $10 \%$ to $15 \%$ are closed or covered by skin. Spina bifida is compatible with survival, although in most cases individuals have moderate to severe disabilities and, in some cases, may have mental retardation $[2,5]$. Researchers generally agreed that NTD occurrence is caused by genetic and environmental factors, though its etiology is not fully understood. Folic acid effectively reduced NTD, and this is supported by evidence from recent systematic reviews on folic acid for the prevention of NTDs in both high- and low-income countries [6,7]. Results of randomized controlled trials and several observational 
Page 2 of 8

studies showed that $50 \%$ to $85 \%$ of NTDs can be prevented if women consume a folic acid-containing supplement before and during the early weeks of pregnancy in addition to dietary folate [1].

Although folic acid supplementation effectively reduces NTD risk, its mechanism is not fully clear. According to the folic acid metabolic pathways [8], two effects of folic acid supplementation protect people from NTDs: (1) it ensures adequate DNA synthesis, methylation, and chromosome repair [9]; and (2) it eliminates the toxic effects of homocysteine to the fetus through the cysteine metabolic pathway [10]. Epidemiologic evidence linking genetic susceptibility in folate metabolism to birth defects is most extensive for NTDs. Genes involved in folate metabolism are heavily investigated for NTD genetic risk, including 5, 10-methylenetetrahydrofolate reductase (MTHFR), Cystathionine beta-synthase (CBS), and Methyltetrahydrofolatehomocysteine methyltransferase (MTR). Also, single nucleotide polymorphisms (SNPs) located in MTHFR, CBS, and MTR have been investigated for their association with NTDs, such as MTHFR-C677T, -A1298C, CBS-T833C, and MS-A2756G. However, findings on these genetic variants have not been consistent [11-15]. Also, few reports exist on other genes involved in the folate metabolic pathway. In this report, we detected 12 SNPs distributed in 11 folate metabolism genes using $\mathrm{SNaPShot}$ genotyping technology and analyzed the association between SNPs involved in folate metabolism and NTDs.

\section{Materials and Methods}

\section{Data sources}

We enrolled 264 subjects in this study with their informed consent, including 76 cases of NTD babies and 188 normal control babies born in the Suzhou Maternal-child Medical Center from 2008 to 2011. The case group included 76 infants diagnosed with NTDs in the Nanjing Medical University Affiliated Suzhou Hospital and Suzhou University Affiliated Children Hospital from 2008 to 2011, and the information of gender, age and diagnosis was shown in Table 1. The control group included 188 healthy infants born in the Nanjing Medical University Affiliated Suzhou Hospital during the same period. They were randomly selected. All babies were Han Chinese, and we collected their umbilical cord blood when they were born.

\begin{tabular}{|l|l|l|l|}
\hline Groups & Gender & Age & Number \\
\hline \multirow{2}{*}{ Spina bifida } & Male & $0 \sim 3$ & 36 \\
\cline { 2 - 4 } & Female & $0 \sim 5$ & 25 \\
\hline \multirow{2}{*}{ Anencephaly } & Male & 0 & 5 \\
\cline { 2 - 4 } & Female & 0 & 10 \\
\hline \multirow{2}{*}{ Total } & Male & $0 \sim 3$ & 41 \\
\cline { 2 - 4 } & Female & $0 \sim 5$ & 35 \\
\hline
\end{tabular}

Table 1: The information of 76 babies with NTD registered.

\section{Genotyping method}

We detected 12 SNPs distributed in 11 folate metabolic enzyme genes in one reaction using SNaPShot genotyping technology. We modified SNaPShot protocol [16] and confirmed it using Sanger sequencing established in our laboratory. The primers (with patents) were synthesized by the Genearray Company in China.

\section{Statistical analysis}

We analyzed the genotypes frequencies between the two groups using the $\mathrm{X}^{2}$-test, and Hardy-Weinberg genetic equilibrium were tested by non-parametric test. We used binary logistic regression analysis to analyze the association between SNPs and NTD, and to remove the influence of other factors, Values of $P<0.05$ were considered statistically significant.

\section{Results}

\section{Genotype distribution}

For the case and control groups, the 12 SNP genotype frequency distributions are shown in Table 2. There were 11 SNPs, including DHFR-c594 + 59del19, GST01-C428T, MTHFD-G1958A, MTHFRC677T, -A1298C, MTR-A2756G, MTRR-A66G, NFE2L2-ins1 + C11108T, RFC1-G80A, TCN2-C776T, and TYMS-1494del6, which had three kinds of alleles: wild-type (- / -), heterozygous mutations $(-/+)$, and homozygous mutations $(+/+)$. CBS-C699T had two genotypes of wild-type $(-/-)$ and heterozygous mutations $(-/+)$. We tested that the genotype frequencies of the 12 SNPs were coincidental with HardyWeinberg genetic equilibrium [16]. The TT homozygous genotype frequency of TYMS-1494del6 was $25.0 \%$ in the NTD group, significantly lower than $47.3 \%$ in the control group. The resulting OR for NTDs carrying the TT homozygous genotype of TYMS-1494del6 compared to the control group was $0.24,95 \% \mathrm{CI}=[0.11-0.54]$, $P<0.001$. Although the AT heterozygous genotype of TYMS-1494del6 in the NTD group was lower than in control group, there was no statistical significance, $P>0.05$. The AT+TT genotype of TYMS-1494del6 in the NTD group was 77.6\%, significantly lower than $89.9 \%$ in the control group. The resulting OR for NTDs carrying the AT+TT genotype of TYMS-1494del6 compared to the control group was $0.39,95 \% \mathrm{CI}=[0.19-0.80], P=0.009$. The homozygous genotype and heterozygous genotype frequencies of 11 SNPs had no significant differences between the NTD and control groups, though 5 SNPs were lower in the NTD group, including MTHFR-C677T, MTRR-A66G, CBS-C699T, GSTO1-C428T, and DHFR-c594+59del19. The 6 SNPs were higher in the NTD group, including MTHFD-G1958A, MTRA2756G, NFE2L2-ins1+C11108T, RFC1-G80A, MTHFR-A1298C, and TCN2-C776G. We used binary logistic regression to analyze the associations between 12 SNPs and NTDs (Table 3). The G allele of TCN2-C776G was a risk factor for NTDs, RR $=1.92, P<0.05$. However, the $\mathrm{T}$ allele of TYMS-1494del6 was a protective factor for NTDs, $\mathrm{RR}=0.45, \mathrm{P}<0.05$. The other SNP alleles were not significant factors for NTDs.

\begin{tabular}{|l|l|l|l|l|l|l|}
\hline SNPs & Genotype & $\begin{array}{l}\text { Control } \\
\mathbf{n = 1 8 8}\end{array}$ & $\begin{array}{l}\text { NTD } \\
\text { N=76 }\end{array}$ & OR & $\mathbf{9 5 \%}$ Cl & P values \\
\hline MTHFD-G1958A(rs2236225) & GG & $109(58.0 \%)$ & $42(55.3 \%)$ & -- & -- & -- \\
\hline
\end{tabular}


Citation: Zhu Q, Li L, Wang T, Jiang W, Ding J, et al. (2016) Genetic Study of 12 SNPs involved in 11 Folate Metabolism Genes and Neural Tube Defects in Suzhou Children. J Mol Genet Med 10: 219. doi:10.4172/1747-0862.1000219

Page 3 of 8

\begin{tabular}{|c|c|c|c|c|c|c|}
\hline & GA & $64(34.0 \%)$ & $30(39.5 \%)$ & 1.22 & $0.69-2.13$ & 0.493 \\
\hline & AA & $15(8.0 \%)$ & $4(5.3 \%)$ & 0.69 & $0.22-2.21$ & 0.532 \\
\hline & $\mathrm{GA}+\mathrm{AA}$ & $79(42.0 \%)$ & $34(44.7 \%)$ & 1.12 & $0.65-1.91$ & 0.686 \\
\hline \multirow[t]{4}{*}{ MTHFR-C677T(rs1801133) } & $\mathrm{CC}$ & $53(28.2 \%)$ & $25(32.9 \%)$ & -- & -- & -- \\
\hline & CT & $100(53.2 \%)$ & $35(46.1 \%)$ & 0.74 & $0.40-1.37$ & 0.338 \\
\hline & TT & $35(18.6 \%)$ & $16(21.1 \%)$ & 0.97 & $0.45-2.07$ & 0.935 \\
\hline & $\mathrm{CT}+\mathrm{TT}$ & $135(71.8 \%)$ & $51(67.1 \%)$ & 0.80 & $0.45-1.42$ & 0.448 \\
\hline \multirow[t]{4}{*}{ MTR-A2756G(rs1805087) } & AA & $153(81.4 \%)$ & $58(76.3 \%)$ & -- & -- & -- \\
\hline & $A G$ & $33(17.6 \%)$ & $18(23.7 \%)$ & 1.44 & $0.75-2.75$ & 0.270 \\
\hline & GG & $2(1.1 \%)$ & $0(0.0 \%)$ & -- & -- & -- \\
\hline & $A G+G G$ & $35(18.6 \%)$ & $18(23.7 \%)$ & 1.36 & $0.71-2.58$ & 0.352 \\
\hline \multirow[t]{4}{*}{ NFE2L2-ins1+C11108T(rs1806649) } & $\mathrm{CC}$ & $163(86.7 \%)$ & $65(85.5 \%)$ & -- & -- & -- \\
\hline & CT & $23(12.2 \%)$ & $10(13.2 \%)$ & 1.09 & $0.49-2.42$ & 0.831 \\
\hline & TT & $2(1.1 \%)$ & $1(1.3 \%)$ & 1.25 & $0.11-14.07$ & 0.854 \\
\hline & $\mathrm{CT}+\mathrm{TT}$ & $25(13.3 \%)$ & $11(14.5 \%)$ & 1.10 & $0.51-2.37$ & 0.801 \\
\hline \multirow[t]{4}{*}{ MTRR-A66G(rs1801394) } & AA & $105(55.9 \%)$ & $46(60.5 \%)$ & -- & -- & -- \\
\hline & $A G$ & $71(37.8 \%)$ & $27(35.5 \%)$ & 0.87 & $0.49-1.52$ & 0.622 \\
\hline & GG & $12(6.4 \%)$ & $3(3.9 \%)$ & 0.57 & $0.15-2.11$ & 0.397 \\
\hline & $A G+G G$ & $83(44.1 \%)$ & $30(39.5 \%)$ & 0.83 & $0.48-1.42$ & 0.487 \\
\hline \multirow[t]{4}{*}{ CBS-C699T(rs234706) } & $\mathrm{CC}$ & $175(93.1 \%)$ & $72(94.7 \%)$ & -- & -- & -- \\
\hline & CT & $13(6.9 \%)$ & $4(5.3 \%)$ & 0.75 & $0.24-2.37$ & 0.621 \\
\hline & TT & $0(0.0 \%)$ & $0(0.0 \%)$ & -- & -- & -- \\
\hline & $\mathrm{CT}+\mathrm{TT}$ & $13(6.9 \%)$ & $4(5.3 \%)$ & 0.75 & $0.24-2.37$ & 0.621 \\
\hline \multirow[t]{4}{*}{ RFC1-G80A(rs1051266) } & GG & $52(27.7 \%)$ & $17(22.4 \%)$ & -- & -- & -- \\
\hline & GA & $103(54.8 \%)$ & $41(53.9 \%)$ & 1.22 & $0.63-2.35$ & 0.556 \\
\hline & AA & $33(17.6 \%)$ & $18(23.7 \%)$ & 1.67 & $0.75-3.69$ & 0.204 \\
\hline & $\mathrm{GA}+\mathrm{AA}$ & $136(72.3 \%)$ & $59(77.6 \%)$ & 1.33 & $0.71-2.48$ & 0.376 \\
\hline \multirow[t]{4}{*}{ GSTO1-C428T(rs4925) } & $\mathrm{CC}$ & $127(67.6 \%)$ & $55(72.3 \%)$ & -- & -- & -- \\
\hline & CT & $56(29.8 \%)$ & $21(27.6 \%)$ & 0.87 & $0.48-1.57$ & 0.634 \\
\hline & TT & $5(2.7 \%)$ & $0(0.0 \%)$ & -- & -- & -- \\
\hline & $\mathrm{CT}+\mathrm{TT}$ & $61(32.4 \%)$ & $21(27.6 \%)$ & 0.79 & $0.44-1.43$ & 0.444 \\
\hline \multirow[t]{4}{*}{ MTHFR-A1298C(rs1801131) } & AA & $133(70.7 \%)$ & $49(64.5 \%)$ & & & \\
\hline & $A C$ & $47(25.0 \%)$ & $25(32.9 \%)$ & 1.44 & $0.80-2.59$ & 0.218 \\
\hline & $\mathrm{CC}$ & $8(4.3 \%)$ & $2(2.6 \%)$ & 0.68 & $0.14-3.31$ & 0.629 \\
\hline & $A C+C C$ & $55(29.3 \%)$ & $27(35.5 \%)$ & 1.33 & $0.76-2.34$ & 0.319 \\
\hline DHFR-c594+59del19 & AA & $22(11.7 \%)$ & $13(17.1 \%)$ & -- & -- & -- \\
\hline
\end{tabular}


Citation: Zhu Q, Li L, Wang T, Jiang W, Ding J, et al. (2016) Genetic Study of 12 SNPs involved in 11 Folate Metabolism Genes and Neural Tube Defects in Suzhou Children. J Mol Genet Med 10: 219. doi:10.4172/1747-0862.1000219

Page 4 of 8

\begin{tabular}{|c|c|c|c|c|c|c|}
\hline & $A G$ & $81(43.1 \%)$ & $30(39.5 \%)$ & 0.63 & $0.28-1.40$ & 0.252 \\
\hline & GG & $85(45.1 \%)$ & $33(43.4 \%)$ & 0.66 & $0.30-1.45$ & 0.298 \\
\hline & $A G+G G$ & $166(88.3 \%)$ & $63(82.9 \%)$ & 0.64 & $0.31-1.35$ & 0.241 \\
\hline \multirow[t]{4}{*}{ TCN2-C776G(rs1801198) } & $\mathrm{CC}$ & $36(19.1 \%)$ & $10(13.2 \%)$ & -- & -- & -- \\
\hline & GC & $100(53.2 \%)$ & $41(53.9 \%)$ & 1.48 & $0.67-3.25$ & 0.332 \\
\hline & GG & $\begin{array}{l}52 \\
(22597.7 \%)\end{array}$ & $25(32.9 \%)$ & 1.73 & $0.74-4.04$ & 0.202 \\
\hline & $G C+G G$ & $152(80.9 \%)$ & $66(86.8 \%)$ & 1.56 & $0.73-3.34$ & 0.245 \\
\hline \multirow[t]{4}{*}{ TYMS-1494del6 } & AA & $19(10.1 \%)$ & $17(22.4 \%)$ & -- & -- & -- \\
\hline & AT & $81(43.1 \%)$ & $40(52.6 \%)$ & 0.56 & $0.26-1.19$ & 0.129 \\
\hline & TT & $88(46.8 \%)$ & $19(25.0 \%)$ & 0.24 & $0.11-0.54$ & $<0.001 \ddagger$ \\
\hline & $\mathrm{AT}+\mathrm{TT}$ & 169 (89.9\%) & $59(77.6 \%)$ & 0.39 & $0.19-0.80$ & $0.009 \ddagger$ \\
\hline
\end{tabular}

Table 2: Comparison of genotype distributions of 12 SNPs in NTD and control babies. $†$ P value of overall association based upon X2-test. $\ddagger$ Cases with significant difference.

\begin{tabular}{|l|l|l|l|l|l|l|}
\hline SNPS & B & S.E. & Wald & df & P & OR \\
\hline MTHFD-G1958A & 0.106 & 0.238 & 0.199 & 1 & 0.656 & 1.112 \\
\hline MTHFR-C677T & 0.109 & 0.227 & 0.232 & 1 & 0.630 & 1.116 \\
\hline MTR-A2756G & 0.008 & 0.346 & 0.001 & 1 & 0.980 & 1.009 \\
\hline NFE2L2-ins1C11108T & 0.057 & 0.373 & 0.023 & 1 & 0.879 & 1.058 \\
\hline MTRR-A66G & -0.404 & 0.257 & 2.468 & 1 & 0.116 & 0.668 \\
\hline CBS-C699T & -0.087 & 0.640 & 0.019 & 1 & 0.891 & 0.916 \\
\hline RFC1-G80A & 0.320 & 0.220 & 2.106 & 1 & 0.147 & 1.377 \\
\hline GST01-C428T & -0.214 & 0.295 & 0.527 & 1 & 0.468 & 0.807 \\
\hline MTHFR-A1298C & 0.272 & 0.289 & 0.881 & 1 & 0.348 & 1.312 \\
\hline DHFR-c59459del19 & -0.143 & 0.211 & 0.459 & 1 & 0.498 & 0.867 \\
\hline TCN2-C776G & 0.655 & 0.220 & 8.854 & 1 & 0.003 & 1.924 \\
\hline TYMS-1494del6 & -0.809 & 0.221 & 13.385 & 1 & 0.000 & 0.445 \\
\hline Constant & -0.745 & 0.579 & 1.658 & 1 & 0.198 & 0.475 \\
\hline
\end{tabular}

Table 3: Binary logistic analysis of the independent effects of 12 SNPs on NTDs. B: Regression coefficient; S.E.: Standard error; Wald: the chisquare value of Wald tests; df: degree of free; OR: odd ratio.

\section{Compound mutation genotypes}

NTDs result from multiple genes interacting, and one gene defect tends to have a tiny effect on NTDs. Compound mutation genotypes were analyzed as one risk factor for NTD. We identified only 3 compound mutation genotypes, showing a significant difference between NTD and control babies (Table 4). The compound mutation genotypes including MTHFD-G1958A, MTHFR-C677T, and MTRA2756G, and $M T H F D-G 1958 \mathrm{~A}, M T R-\mathrm{A} 2756 \mathrm{G}$, and RFC1-G80A were
9.2\% in NTD babies, significantly higher than $2.7 \%$ in control babies, suggesting that two genotypes were risk factors for NTD, $\mathrm{OR}=3.71$, 95\% CI=1.14-12.09, $P=0.021$. Additionally, the compound mutation genotype of RFC1-G80A, MTHFR-A1298C, and TCN2-C776T was $25.0 \%$ in NTD babies, significantly higher than $13.8 \%$ in control babies, suggesting it was also risk factor for NTDs, OR=2.07, 95\% $\mathrm{CI}=1.07-4.04, P=0.029$. 


\begin{tabular}{|c|c|c|c|c|c|c|}
\hline & \multirow[t]{2}{*}{ Groups } & \multicolumn{2}{|c|}{ Compound mutation genotypes } & \multirow[t]{2}{*}{ OR } & \multirow[t]{2}{*}{$95 \% \mathrm{Cl}$} & \multirow[t]{2}{*}{$\mathbf{P}$} \\
\hline & & YES & NO & & & \\
\hline \multirow[t]{2}{*}{ MTHFD-G1958A, MTHFR-C677T and MTR-A2756G } & NTD & 7 & 69 & \multirow[t]{2}{*}{3.71} & \multirow[t]{2}{*}{$1.14-12.09$} & \multirow[t]{2}{*}{0.021} \\
\hline & Control & 5 & 183 & & & \\
\hline \multirow[t]{2}{*}{ MTHFD-G1958A, MTR-A2756G and RFC1-G80A } & NTD & 7 & 69 & \multirow[t]{2}{*}{3.71} & \multirow[t]{2}{*}{$1.14-12.09$} & \multirow[t]{2}{*}{0.021} \\
\hline & Control & 5 & 183 & & & \\
\hline \multirow[t]{2}{*}{ RFC1-G80A, MTHFR-A1298C TCN2-C776T } & NTD & 19 & 57 & \multirow[t]{2}{*}{2.07} & \multirow[t]{2}{*}{$1.07-4.04$} & \multirow[t]{2}{*}{0.029} \\
\hline & Control & 26 & 162 & & & \\
\hline
\end{tabular}

Table 4: Comparison of compound mutation genotypes in NTD and control babies.

\section{Compound wide-type genotypes}

No compound wild-type genotypes showed a significant difference in NTD and control babies except the compound wild-type genotype RFC1-G80A, MTHFR-A1298C, and TCN2-C776T (Table 5). Fourteen control babies carried the compound mutation genotype of the total 188 control babies, but no NTD babies carried this compound mutation. This indicated that the compound wild-type genotype
RFC1-G80A, MTHFR-A1298C, and TCN2-C776T is a protective factor of NTDs, $P=0.014$. Additionally, two compound wild-type genotypes including MTHFD-G1958A, MTHFR-C677T, and MTRA2756G, and MTHFD-G1958A, MTR-A2756G, and RFC1-G80A may be protective NTD factors, with $\mathrm{OR}=0.73$ and 0.61 , respectively, although they showed no significant differences given our small sample size.

\begin{tabular}{|c|c|c|c|c|c|c|}
\hline & \multirow[t]{2}{*}{ Groups } & \multicolumn{2}{|c|}{ Compound wide-type genotypes } & \multirow[t]{2}{*}{ OR } & \multirow[t]{2}{*}{$95 \% \mathrm{Cl}$} & \multirow[t]{2}{*}{$\mathbf{P}$} \\
\hline & & YES & NO & & & \\
\hline \multirow[t]{2}{*}{ MTHFD-G1958A, MTHFR-C677T, and MTR-A2756G } & NTD & 8 & 68 & \multirow[t]{2}{*}{0.73} & \multirow[t]{2}{*}{$0.32-1.70$} & \multirow[t]{2}{*}{0.468} \\
\hline & Control & 26 & 162 & & & \\
\hline \multirow[t]{2}{*}{ MTHFD-G1958A, MTR-A2756G, and RFC1-G80A } & NTD & 6 & 70 & \multirow[t]{2}{*}{0.61} & \multirow[t]{2}{*}{$0.24-1.58$} & \multirow[t]{2}{*}{0.307} \\
\hline & Control & 23 & 165 & & & \\
\hline \multirow[t]{2}{*}{ RFC1-G80A, MTHFR-A1298C, and TCN2-C776T } & NTD & 0 & 76 & \multirow[t]{2}{*}{--} & \multirow[t]{2}{*}{--} & \multirow[t]{2}{*}{0.014} \\
\hline & Control & 14 & 174 & & & \\
\hline
\end{tabular}

Table 5: Comparison of compound wild-type genotypes in NTD and control babies.

\section{Discussion}

There were several interesting opinions revealed in this study. We found the TT and AT of TYMS-1494del6 were the protective NTD factors, with $\mathrm{OR}=0.39$ and 0.56 respectively. Furthermore, TYMS-1494del6 was also a protective NTD factor as it removed the effects of other SNPs, RR=0.445, $P<0.001$. TYMS was the key enzyme in the de novo synthesis of 29-deoxyuridine -59-monophosphate (dTMP), the essential precursor of DNA biosynthesis and the repairing process [17]. The most common deletion polymorphism of TYMS was 1494del6 in intron-1, which might have played a more associative role in regulating gene expression than the promoter [17]. At present, two studies were carried out on the association between the TYMS gene SNPs and NTDs, and the results indicated that several TYMS gene SNPs could have been NTD risk factors $[18,19]$. However, no study reported the association between TYMS-1494del6 and NTDs, and our results indicated it was an interesting candidate SNP for NTDs.

We found another candidate SNP for NTDs was TCN2-C776T. When it removed the effects from other SNPs, TCN2-C776T could be an NTD risk factor, with $\mathrm{RR}=1.924, P=0.003$. TCN's main function was transporting VB12 into cells, critical for balancing folate metabolism. The most common polymorphism was TCN2-C776T, which encoded for arginine in place of proline at codon 259 of the amino acid sequence [20]. Several studies reported TCN-C776G was associated with NTD [21-24]. To a certain extent, our results supported the above conclusion.

The association of SNPs localized at MTHFR gene with NTD risk was the research focus. We investigated the distribution of MTHFRC677T and -A1298C in NTDs and could not conclude that they were NTD genetic risk factors, which contrasted with most of the other researchers' conclusions $[15,25]$. The cause of the contradictory results may include that since NTDs are caused by the combined effects of many genes, different genetic backgrounds and nutrition may result in different genetic NTD susceptibility. MTHFD1-G1958A was a common polymorphism, resulting in a substitution of alanine for glycine at codon 653 , located at the domain 10formylTHF enzyme synthase. The purified Arg653Gln enzyme had normal substrate affinity, but the mutation reduced MTHFD1's metabolic activity 
[26,27]. Several studies reported that MTHFD1-G1958A could be a maternal NTD risk factor [28,29], but not a children factor. However, De Marco et al. [30] found an increased risk for heterozygous 1958G/A and homozygous 1958A/A genotypes in children. Our results also indicated a negative association between MTHFD1-G1958A and NTDs, consistent with conclusions from studies by Parle-McDermott and Brody.

The most common polymorphism in the MTR gene was MTRA2756G, which decreased MTR activity and increased the cellular homocysteine level [31,32]. To date, numerous studies have reported that an association exists between the MTR-A2756G polymorphism and maternal NTD risk. However, their results remain inconsistent [33]. The latest meta-analysis by Ouyang indicated that the MTRA2756G polymorphism was significantly associated with maternal NTD risk in Caucasians [34] but not associated with NTD risks in Caucasian children [34]. According to our studies, MTR-A2756G was not associated with NTD risks in Chinese children.

MTRR is a member of the electron transferase family with three characteristic binding sites, including FMN, FAD, and NADPH Disturbances in catalytic activity can lead to high homocysteine levels, which could be an NTD risk factor [22]. The most common polymorphism in the MTRR gene was A66G substitution, leading to isoleucine changing to methionine in amino acid 22. Therefore, the association between $M T R R$-A66G and NTDs has been extensively researched. Pietrzyk et al. [35] postulated that MTRR-A66G should be regarded as an independent risk factor for spina bifida. Also, Dunlevy found that the 66GG genotype frequency is significantly higher in people with NTDs and their mothers than in the control group [36] However, most studies, including the meta-analysis, indicated MTRRA66G was not associated with NTD risk [34], In Chinese children, $M T R R$-A66G heterozygous and homozygous genotype were not shown a association with NTDs.

CBS catalyzes homocysteine and serine to synthesize irreversible cystathionine. Disturbances in this process could lead to an increased cellular homocysteine level. CBS is a cytoplasmic protein and has a catalytic core with a conserved amino acid sequence. In addition, 60 percent of about 150 mutations could lead to decreased protein catalytic activity. There are three polymorphisms in $C B S$, including $C B S$-844ins68bp, $C B S$-T833C, and $C B S$-G919A, reported as genetic factors for CHDs [37] and NTDs [18]. In our study, we did not find that a novel SNP CBS-C699T was the NTD risk factor.

The most common polymorphism in the RFC1 gene is a highfrequency $G$ to $A$ SNP at position 80 that results in a change of arginine-27 to histidine-27 in exon 2. This makes 5- formyl tetrahydrofolate cofactor transport 2-fold lower than the wildtype, because the arginine (CGG) at residue 27 could be critical for targeting and integrating protein to the plasma membrane. Many studies showed that women carrying the GA and AA genotypes had higher RBC folate concentrations relative to women carrying the RFC1-80GG genotype, and this was an independent risk factor for their children with CHD [16]. However, our results did not support the above conclusion. The possible reason may have been that folate metabolism is a complicated metabolic process in which many enzymes play important roles of maintaining metabolism. Due to the different genetic backgrounds in our study, the major gene defects leading to elevated homocysteine were different.

DHFR's continuous reduction of DHF to tetrahydrofolates (THFs) played an important role in folate metabolism. The most common polymorphism in the DHFR gene was a 19bp deletion within intron I, a well known site of regulatory sequences for some genes. Three studies reported the association between DHFR-c594+59del19 and NTDs but did not reach a consistent conclusion [38-40]. In this study, we did not find that NTDs were correlated with DHFR-c594+59del19.

We first reported the association between two SNPs, including NFE2L2-ins1C11108T and GST01-C428T, and NTDs. NFE2L2 was a transcription factor regulating antioxidant enzyme protein expression at the transcriptional level, and plays an important role in the process of HCY metabolism. GSTO1 played an important role in the metabolic pathway from homocysteine to glutathione, which is referred as the transsulfuration pathway. Mostly polymorphisms of GSTM1, GSTT1, and GSTP1 were revealed to be associated with increased susceptibility to several cancers and other diseases [41,42]. In this study, NFE2L2ins1C11108T and GST01-C428T were not considered NTD risk factors.

\section{Conclusion}

The vast majority of NTDs resulted from the interaction of multiple genes and the environment. The effect of one SNP or gene tended to be too "minor" to be found. Also, combined SNPs effects on NTDs may be more obvious than simple superposition of SNPs. However, few studies focused on the association between NTDs and the compound mutations of several significant SNPs located on enzyme genes involved in folate metabolism. We further analyzed the combined effects of SNPs in multiple folate metabolism enzyme genes on NTDs. We found that three compound mutation genotypes, including MTHFD-G1958A, MTHFR-C677T, and MTR-A2756G; MTHFDG1958A, MTR-A2756G, and RFC1-G80A; and RFC1-G80A, MTHFRA1298C and TCN2-C776T could be considered risk factors for NTDs. Recently, the two compound mutation genotypes of $M T H F D-G 1958$ A, MTHFR-C677T, and MTR-A2756G, and MTHFD-G1958A, MTRA2756G, and RFC1-G80A were also reported as CHD risk factors by Wang $B$, et al. which also indicated the compound mutation genotype should be considered an independent factor. The compound wide-type genotypes of MTHFD-G1958A, MTHFR-C677T, and MTR-A2756G, and MTHFD-G1958A, MTR-A2756G, and RFC1-G80A could be protective factors for NTD, with $\mathrm{OR}<1$. However, the differences between the two compound wide-type genotypes did not reach statistical significance, $P>0.05$, which may be due to our small sample size. The compound mutation genotype of RFC1-G80A, MTHFRA1298C, and TCN2-C776T was another genetic factor for NTDs, $\mathrm{OR}=2.07, \quad P=0.029$. Furthermore, we found evidence that the compound wide-type genotype of RFC1-G80A, MTHFR-A1298C, and TCN2-C776T was a protective factor for NTDs. We found that 14 control babies carried the compound wide-type genotype of RFC1G80A, MTHFR-A1298C, and TCN2-C776T, but not in NTD babies.

Limited by the sample size and SNPs detected in our studies, we could not analyze and screen all compound genotypes. Therefore, further studies with large sample sizes and more SNPs related to folate metabolism are needed to research NTDs. High-throughput genotyping technologies have superiority in detecting SNPs, such as Gene Chip Technique, Genome-wide Association Analysis, and Second-generation Sequencing Technology, which may be the breakthroughs for exploring NTD genetic risk factors. 


\section{Acknowledgement}

We thank the patients for participating in this research project. This work is supported by clinical medicine science and technology projects of Jiangsu province (BL2013019), Suzhou Key Medical Center (Szzx201505) and Natural Science Foundation of Ningxia Autonomous Region (NZ16226).

\section{References}

1. Fonseca EB, Raskin S, Zugaib M (2013) Folic acid for the prevention of neural tube defects. Rev Bras Ginecol Obstet 35: 287-289.

2. Yi Y, Lindemann M, Colligs A, Snowball C (2011) Economic burden of neural tube defects and impact of prevention with folic acid: a literature review. Eur J Pediatr 170: 1391-1400.

3. Lin S, Herdt-Losavio ML, Chapman BR, Munsie JP, Olshan AF, et al (2013) Maternal occupation and the risk of major birth defects: a followup analysis from the National Birth Defects Prevention Study. Int J Hyg Environ Health 216: 317-323.

4. Au KS, Ashley-Koch A, Northrup H (2010) Epidemiologic and genetic aspects of spina bifida and other neural tube defects. Dev Disabil Res Rev 16: 6-15.

5. Toriello HV (2005) Folic acid and neural tube defects. Genet Med 7: 283-284.

6. Blencowe H, Cousens S, Modell B, Lawn J (2010) Folic acid to reduce neonatal mortality from neural tube disorders. Int J Epidemiol 39: i110i121.

7. Wolff T, Witkop CT, Miller T, Syed SB (2009) Folic acid supplementation for the prevention of neural tube defects: an update of the evidence for the U.S. Preventive Services Task Force. Ann Intern Med 150: 632-639.

8. Lim U, Wang SS, Hartge P, Cozen W, Kelemen LE, et al. (2007) Genenutrient interactions among determinants of folate and one-carbon metabolism on the risk of non-Hodgkin lymphoma: NCI-SEER casecontrol study. Blood 109: 3050-3059.

9. Friso S, Choi SW, Girelli D, Mason JB, Dolnikowski GG, et al. (2002) A common mutation in the 5,10-methylene tetrahydrofolate reductase gene affects genomic DNA methylation through an interaction with folate status. Proc Natl Acad Sci USA 99: 5606-5611.

10. Özer I, Özçetin M, Karaer H, Kurt SG, Şahin Ş (2011) Retrospective approach to methylene tetrahydrofolate reductase mutations in children. Pediatr Neurol 45: 34-38.

11. Yang Y, Chen J, Wang B, Ding C, Liu H (2015) Association between MTHFR C677T polymorphism and neural tube defect risks: A comprehensive evaluation in three groups of NTD patients, mothers, and fathers. Birth Defects Res A Clin Mol Teratol 103: 488-500.

12. Koch MC, Stegmann K, Ziegler A, Schröter B, Ermert A (1998) Evaluation of the MTHFR C677T allele and the MTHFR gene locus in a German spina bifida population. Eur J Pediatr 157: 487-92.

13. Morrison K, Papapetrou C, Hol FA, Mariman EC, Lynch SA, et al. (1998) Susceptibility to spina bifida; an association study of five candidate genes. Ann Hum Genet 62: 379-396.

14. Ouyang S, Liu Z, Li Y, Ma F, Wu J (2014) Cystathionine beta-synthase 844 ins68 polymorphism is unrelated to susceptibility to neural tube defects. Gene 535: 119-123.

15. Gonzalez-Herrera L, Castillo-Zapata I, Garcia-Escalante G, PintoEscalante (2007) DA1298C polymorphism of the MTHFR gene and neural tube defects in the state of Yucatan, Mexico. Birth Defects Res A Clin Mol Teratol 79: 622-626.

16. Wang B, Liu M, Yan W, Mao J, Jiang D, et al. (2013) Association of SNPs in genes involved in folate metabolism with the risk of congenital heart disease. J Matern Fetal Neonatal Med 26: 1768-1777.

17. Kim SR, Ozawa S, Saito Y, Kurose K, Kaniwa N, et al. (2006) Fourteen novel genetic variations and haplotype structures of the TYMS gene encoding human thymidylate synthase (TS). Drug Metab Pharmacokinet 21: 509-516
18. Shaw GM, Lu W, Zhu H, Yang W, Briggs FBS, et al. (2009) 118 SNPs of folate-related genes and risks of spina bifida and conotruncal heart defects. BMC Med Genet 10: 49.

19. Volcik KA, Shaw GM, Zhu H, Lammer EJ, Laurent C, et al. (2003) Associations between polymorphisms within the thymidylate synthase gene and spina bifida. Birth Defects Res A Clin Mol Teratol 67: 924-928.

20. Namour F, Guy M, Aimone-Gastin I, de Nonancourt M, Mrabet N, et al. (1998) Isoelectrofocusing phenotype and relative concentration of transcobalamin II isoproteins related to the codon 259 Arg/Pro polymorphism. Biochem Biophys Res Commun 251: 769-774.

21. Afman LA, Lievers KJ, van der Put NM, Trijbels FJ, Blom HJ (2002) Single nucleotide polymorphisms in the transcobalamin gene: relationship with transcobalamin concentrations and risk for neural tube defects. Eur J Hum Genet 10: 433-438.

22. Guéant-Rodriguez RM, Rendeli C, Namour B, Venuti L, Romano A, et al (2003) Transcobalamin and methionine synthase reductase mutated polymorphisms aggravate the risk of neural tube defects in humans. Neurosci Lett 344: 189-192.

23. Martinelli M, Scapoli L, Palmieri A, Pezzetti F, Baciliero U, et al. (2006) Study of four genes belonging to the folate pathway: transcobalamin 2 is involved in the onset of non-syndromic cleft lip with or without cleft palate. Hum Mutat 27: 294.

24. Pietrzyk JJ, Bik-Multanowski M, Sanak M, Twardowska M (2003) Polymorphisms of the 5,10 methylene tetrahydrofolate and the methionine synthase reductase genes as independent risk factors for spina bifida. J Appl Genet 44: 111-113.

25. Greene NDE, Stanier P, Copp AJ (2009) Genetics of human neural tube defects. Hum Mol Genet 18: R113-R129.

26. Silva LM, Silva JN, Galbiatti AL, Succi M, Ruiz MT, et al. (2011) Head and neck carconogenesis: impact of MTHFD1 G1958A polymorphism. Rev Assoc Med Bras 57: 194-199.

27. Christensen KE, Rohlicek CV, Andelfinger GU, Michaud J, Bigras JL, et al. (2009) The MTHFD1 p.Arg653Gln variant alters enzyme function and increases risk for congenital heart defects. Hum Mutat 30: 212-220.

28. Parle-McDermott A, Kirke PN, Mills JL, Molloy AM, Cox C, et al. (2006) Confirmation of the R653Q polymorphism of the trifunctional C1synthase enzyme as a maternal risk for neural tube defects in the Irish population. Eur J Hum Genet 14: 768-772.

29. Brody LC, Conley M, Cox C, Kirke PN, McKeever MP, et al. (2002) A polymorphism, R653Q, in the trifunctional enzyme methylene tetrahydrofolate dehydrogenase/methenyl tetrahydrofolate cyclohydrolase/formyl tetrahydrofolate synthetase is a maternal genetic risk factor for neural tube defects: report of the Birth Defects Research Group. Am J Hum Genet 71: 1207-1215.

30. De Marco P, Merello E, Calevo MG, Mascelli S, Raso A, et al. (2006) Evaluation of a methylene tetrahydrofolate-dehydrogenase $1958 \mathrm{G}>\mathrm{A}$ polymorphism for neural tube defect risk. J Hum Genet 51: 98-103.

31. van der Put NM, van der Molen EF, Kluijtmans LA, Heil SG, Trijbels JM, et al. (1997) Sequence analysis of the coding region of human methionine synthase: relevance to hyperhomocysteinaemia in neural-tube defects and vascular disease. QJM 90: 511-517.

32. Chen LH, Liu ML, Hwang HY, Chen LS, Korenberg J, et al. (1997) Human methionine synthase. cDNA cloning, gene localization, and expression. J Biol Chem 272: 3628-3634.

33. Ouyang S, Li Y, Liu Z, Chang H, Wu J (2013) Association between MTR A2756G and MTRR A66G polymorphisms and maternal risk for neural tube defects: a meta-analysis. Gene 515: 308-312.

34. Ouyang S, Liu Z, Li Y, Wu J (2013) Meta-analyses on the association of MTR A2756G and MTRR A66G polymorphisms with neural tube defect risks in Caucasian children. J Matern Fetal Neonatal Med 26: 1166-1170.

35. Pietrzyk JJ, Bik-Multanowski M (2003) 776C >G polymorphism of the transcobalamin II gene as a risk factor for spina bifida. Mol Genet Metab 80: 364 .

36. Dunlevy LP, Chitty LS, Burren KA, Doudney K, Stojilkovic-Mikic T, et al. (2007) Abnormal folate metabolism in foetuses affected by neural tube defects. Brain 130: 1043-1049. 
Citation: Zhu Q, Li L, Wang T, Jiang W, Ding J, et al. (2016) Genetic Study of 12 SNPs involved in 11 Folate Metabolism Genes and Neural Tube Defects in Suzhou Children. J Mol Genet Med 10: 219. doi:10.4172/1747-0862.1000219

Page 8 of 8

37. Song XM, Zheng XY, Zhu WL, Huang L, Li Y (2006) Relationship between polymorphism of cystathionine beta synthase gene and congenital heart disease in Chinese nuclear families. Biomed Environ Sci 19: $452-456$

38. Johnson WG, Stenroos ES, Spychala JR, Chatkupt S, Ming SX, et al. (2004) New 19 bp deletion polymorphism in intron-1 of dihydrofolate reductase (DHFR): a risk factor for spina bifida acting in mothers during pregnancy? Am J Med Genet A 124A: 339-345.

39. Parle-McDermott A, Pangilinan F, Mills JL, Kirke PN, Gibney ER, et al. (2007) The 19-bp deletion polymorphism in intron-1 of dihydrofolate reductase (DHFR) may decrease rather than increase risk for spina bifida in the Irish population. Am J Med Genet A 143A: 1174-1180.
40. van der Linden IJ, Nguyen U, Heil SG, Franke B, Vloet S, et al. (2007) Variation and expression of dihydrofolate reductase (DHFR) in relation to spina bifida. Mol Genet Metab 91: 98-103.

41. Konwar R, Manchanda PK, Chaudhary P, Nayak VL, Singh V, et al. (2010) Glutathione S-transferase (GST) gene variants and risk of benign prostatic hyperplasia: a report in a North Indian population. Asian Pac J Cancer Prev 11: 1067-1072.

42. Allen M, Zou F, Chai HS, Younkin CS, Miles R, et al. (2012) Glutathione S-transferase omega genes in Alzheimer and Parkinson disease risk, ageat-diagnosis and brain gene expression: an association study with mechanistic implications. Mol Neurodegener 7: 13 